\title{
Co-simulation for the Design of Controllers in Urban Drainage Systems
}

\author{
G. Riaño-Briceño, A. Ramírez-Jaime, J. Barreiro-Gómez, \\ N. Quijano, Senior Member, IEEE, and C. Ocampo-Martinez, Senior Member, IEEE
}

\begin{abstract}
A co-simulation framework that uses two software tools (i.e., Matlab, Python or LabVIEW, and SWMM) is presented. The co-simulation is performed thanks to a tool that has been developed, and which is the main contribution of this work. This approach uses the storm water management model (SWMM), becoming a solution to the lack of tools to test controllers for urban drainage systems (UDS). Specifically, MatSWMM, an open source framework that can be used to this end, is presented. Additionally, in order to illustrate the features of the co-simulation methodology, some of the issues of using control-oriented models (COM) are pointed out and simulated with MatSWMM, through a simple case study. To this end, and as an illustrative example of the controllers that might be implemented with the proposed tool, a linear model of the system is built and a decentralized population dynamics-based controller is tested. The results obtained show the advantages of the co-simulation tool to evaluate the control performance of these systems.
\end{abstract}

Index Terms-Co-simulation, MatSWMM, Integrated Model of Urban Drainage Systems, Real-Time Control, Population Dynamics-based Control

\section{INTRODUCTION}

The development of integrated models for water systems has become essential in the last decade, because they allow to determine with better precision than simulating independent subsystems the global behavior of urban drainage systems (UDS). However, integration means greater complexity and a higher computational burden. Thus, it can be a challenge since most of the available models today do not consider important components of the system, which may cause critical problems if they are not properly modeled before deployment. For instance, in UDS with sensors and actuators, it can be critical to perform control actions without taking into consideration the communication system failures, or gradually varied flow phenomena that are caused by the movement of gates.

During the last years, several modeling approaches have shown that the design and analysis criteria, despite of being focused on integration, are specialized in the water quality problem [8]. Additionally, most of the studies developed to control UDS are based on control-oriented models (COM), i.e., simplified models that are not holistic at all. The use of holistic models offers great advantages, and several authors

G. Riaño-Briceño, A. Ramírez-Jaime, J. Barreiro-Gómez, and N. Quijano are with Departamento de Ingeniería Eléctrica y Electrónica. G. RiañoBriceño is also with Departamento de Ingeniería Civil, Universidad de los Andes, Carrera $1^{a}$ No 18A-10, Colombia \{ga.riano949, af.ramirez236, j.barreiro135, nquijano\}@uniandes.edu.co

J. Barreiro-Gómez and C. Ocampo-Martinez are with the Automatic Control Department, Universitat Politècnica de Catalunya, Institut de Robòtica i Informàtica Industrial (CSIC-UPC), Llorens i Artigas, 4-6, 08028 Barcelona, Spain. \{jbarreiro, cocampo\}@iri.upc.edu have emphasized this, arguing benefits for the performance [3], optimization of resources [4], and reduction of costs and faults after the commissioning of the system [7].

The main contribution of this paper consists in introducing a new tool to test controllers for UDS called MatSWMM. For this, a simulation-oriented model (SOM) has been adapted to simplify the development of an accurate representation of the run-off dynamics. The model that has been used is the storm water management model (SWMM) of the US-EPA [6]. Its functionality has been enhanced, so that it can be compatible with tools commonly used by control designers. The run-off calculation engine SWMM has been embedded in Matlab, Python, and LabView, and several functions related to system identification and optimization-based controllers have been developed and compiled in MatSWMM. It is an open source framework with two main functionalities: i) efficient modeling of large scale UDS; and ii) automatic generation of COMs for model-based controller design and closed-loop simulation. Furthermore, in order to illustrate the versatility of the proposed co-simulation approach, a controller is designed and implemented with MatSWMM and complementary software tools.

The reminder of the paper is organized as follows. Section II explains how MatSWMM works, its main functionality, and the co-simulation routine. Section III presents a detailed description of the control module that has been included in MatSWMM, for the convergence topology as done in [2]. Section IV introduces an example application of MatSWMM, and Section V shows the results and their brief analysis, emphasizing some problems that cannot be detected with simplified models when implementing real-time control (RTC). Finally, in Section VI, some conclusions are presented and a discussion is made about further applications of the cosimulation by using the novel tool.

\section{The MATSWMM TOOL}

In this section, the main features of MatSWMM are described. First of all, a brief explanation of the SWMM model is done, highlighting the enhanced functionalities that have been adapted to it. Then, each module of MatSWMM related to UDS modeling, system identification and RTC is explained. Finally, some calculation and computational aspects are stated.

\section{A. The SWMM model}

SWMM is a dynamic rainfall-runoff simulation model that is used for planning, analysis, and design of infrastructure related to stormwater run-off, combined and sanitary sewers, 


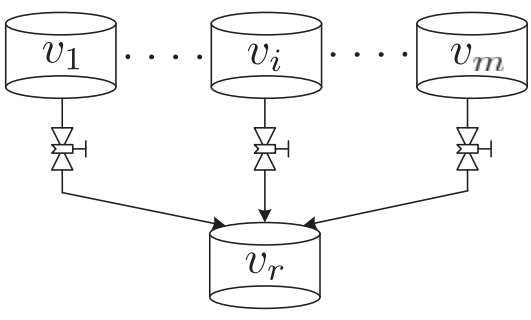

a)

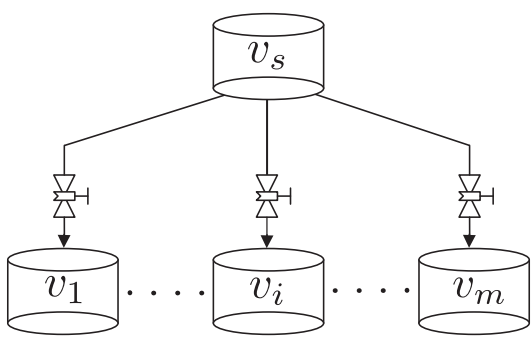

b)

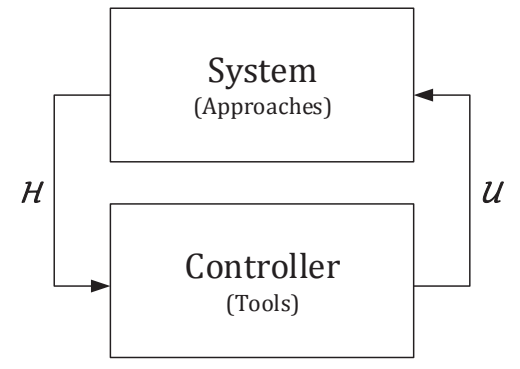

c)

Fig. 1. a) Convergence topology, i.e., $m$ source reservoirs whose outflows converge to a receptor reservoir; b) divergence topology, i.e., a source reservoir whose outflow diverges to $m$ receptor reservoirs; and c) closed-loop scheme with co-simulation.

and other drainage systems in urban areas [6]. The platform consists of an interface where a drainage network can be created, using objects such as pipes, canals, storage units, subcatchments, among others. Additionally, it has a calculation module that uses the one-dimensional Saint-Venant equations (SVE) to simulate run-off throughout the network [13].

The design of sewer systems is experimenting several changes today due to the inclusion of actuators, control rules, and optimal design. For this reason, SWMM has become a limited tool but, it has a big potential as an open source software, since it is possible to enhance its functionality in order to include new methods that allow its users to simulate complex RTC strategies.

Consequently, MatSWMM has been created, bringing new functionality to the program, as it was described before. This framework works as a co-simulation engine that retrieves information from SWMM during the simulation, and allows to modify attributes, transmit control actions, and perform data analysis efficiently. Furthermore, it has been designed to be scalable and easy to use with various programming languages. So far, the framework has been adapted to Matlab, Python, and LabVIEW, using a C dynamic link library (DLL), but it can be extended using the base code ${ }^{1}$.

It is important to emphasize that SWMM is a physicallybased, discrete-time simulation model that uses principles of conservation of energy and momentum [6]. As a discretetime tool, its algorithm finds solutions for the flow-routing problem iteratively. After the calculation of each sub-process in SWMM, the enhanced functionality is executed, i.e., it is possible to retrieve a set of state variables $\mathcal{H}$ and modify parameters during the simulation, through the set of control actions $\mathcal{U}$, as shown in Figure 1c).

\section{B. General description}

As noted above, MatSWMM has been conceived to simplify the process of designing controllers for UDS. Considering that modeling of drainage systems can be distinguished between two groups of models, COM and physically-based models [5], both can be executed with MatSWMM. SWMM is the physically-based model, and the virtual tanks (VT) model

\footnotetext{
${ }^{1}$ The source code and its documentation is availabel at https://github.com/water-systems/MatSWMM.git
}

proposed in [9] has been included as the COM. In order to work with the COM, it is required that the user determines the features of the system, i.e., topology, elements and properties, through the SWMM interface. Then, a state-space representation of the considered system can be retrieved.

Additionally, a default control module has been included in the tool. It is a decentralized population dynamics-based controller, like the one proposed in [2] for the convergence and divergence topologies, which is better explained in Section III. It can be adapted to three of the fundamental dynamics proposed in [12], i.e., the replicator, the projection and the Smith dynamics.

\section{Simulation-oriented model and its functionalities}

For modeling the system, the SWMM default functionalities are used (these are related to its calculation module [6]), which is the one that executes the run-off model using the SVE. These functions are: parameter initialization, running a calculation step, ending sub-processes, calculating the mass balance error, writing a report file, and closing the application. Using these functions the SWMM algorithm [6] can be replicated in Matlab, Python or LabVIEW, with the advantage of having greater control of the whole process.

With that in mind, getters and setters were developed in order to gain an advantage over the partition of the SWMM algorithm. On the one hand, getters are executed every iteration to obtain properties of the model, e.g., flow, velocity, volume, flooding, Froude number, among others. On the other hand, setters are used to modify properties of the model, by far, these allow to adjust the setting of orifices. Thus, it is possible with these functions to enhance the functionality of SWMM, integrating any control strategy with other tools and using gates or orifices as actuators in the system.

Nevertheless, if the model is large, i.e., the number of nodes and conduits is such that it is not possible to retrieve information or modify properties easily, getters and setters become inefficient to manage data. Therefore, there are special methods to handle data using special data structures. Because of the structure of SWMM, graphs are used to fit the model properly. Additionally, when convergence topologies prevail (see Figure 1a)), the structure can be simplified as a tree. The enhanced functionality related to these data structures is 


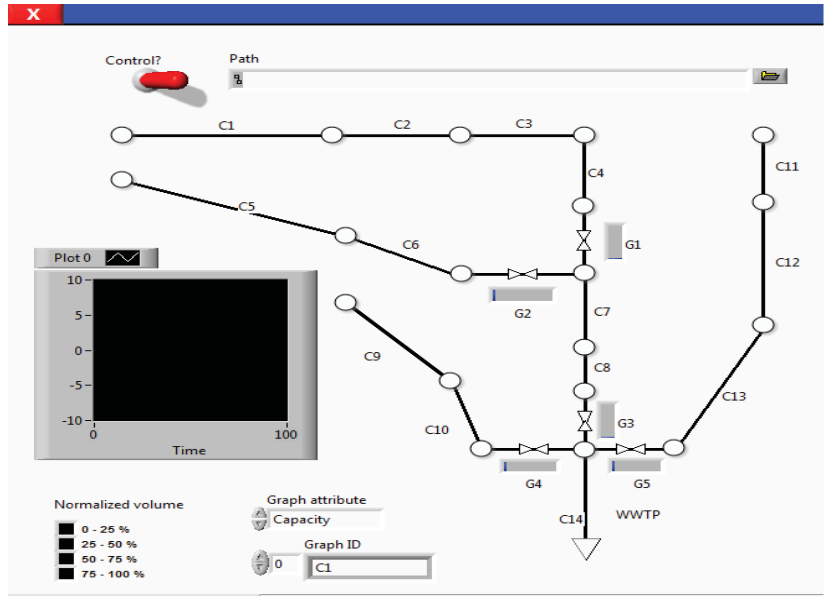

(a)

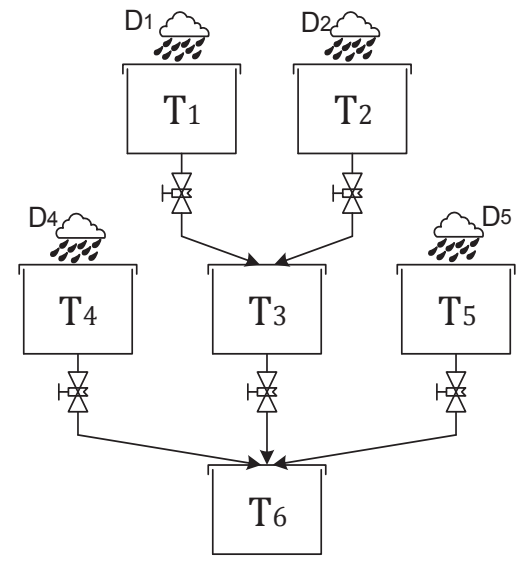

(b)

Fig. 2. Case study; a) divergence case study in MatSWMM for LabVIEW; and b) VT-based equivalent model.

oriented to split the model in manageable pieces. Thus, the process of extracting information and modifying parameters can be done efficiently through traversal algorithms, such as breath-first and depth-first search, which can be useful when implementing a decentralized control strategy.

\section{Computational aspects for system identification}

It is possible to characterize any UDS as a composition of two topologies, i.e., convergence and divergence. As shown in Figure 1a, the convergence topology is related to the case where the flow of several storage units or pipes is merged in a single receptor structure. Furthermore, the divergence topology, shown in Figure 1b, is formed when the flow of a single structure is distributed along more than one receptor. The former one prevails in stormwater UDS while the latter is commonly related to drinking water networks (DWN). However, both topologies are used to built combined sewer networks. In these, not only stormwater is transported, also sanitary and wastewater flows.

MatSWMM has been designed to characterize both topologies easily, guaranteeing a proper modeling of the run-off phenomenon throughout large networks and allowing the user to handle the problem of flow assignation with an optimizationbased controller for each case, as the one described in [2].

The SVE used by SWMM to simulate the run-off throughout the network describe in a quite high level of detail the behavior of the system. Usually, this level of detail is not required in RTC applications and COMs are used instead. As stated before, MatSWMM incorporates the so-called VT-based model, which is a widely used COM for modeling UDS [4][9].

In the virtual reservoir approach, the UDS is divided into a set of interconnected real and virtual tanks (VT). According to [9], a VT is a storage element that represents the total volume of sewage inside the sewer mains associated with a determined portion of a given network. The volume is calculated through the mass balance of the stored volume, the inflows (from both sewage mains and stormwater), and the outflows (to both sewage mains and street) of the reservoir. For the model developed in MatSWMM, the outflow of a given tank (virtual or real) is assumed to be proportional to the volume of the tank. Therefore, the model of a tank is given by $\frac{d V_{i}(t)}{d t}=q_{i}^{i n}(t)-K_{i} V_{i}(t)$, where $V_{i}$ is the volume stored in the $i$-th tank, $q_{i}^{i n}$ is the total inflow to the $i$-th tank, and $K_{i}$ is the volume/flow conversion (VFC) coefficient. As SWMM provides measures for both, the volume and the outflow of the reservoirs, the VFC coefficient for each reservoir can be computed via a least-squares algorithm given by $K_{i}=\arg \min _{K_{i}} \int_{0}^{t_{f}}\left(q_{i}^{\text {out }}(t)-K_{i} V_{i}(t)\right)^{2}$, where $t_{f}$ is the total simulation time, and $q_{i}^{\text {out }}$ is the total outflow of the $i$-th reservoir. Finally, the maximum capacity volume of the $i$-th tank is denoted by $V_{i}^{\max }$.

\section{Control module}

In this section, the MatSWMM decentralized populationdynamics-based control module is described. An explanation of the population dynamics approach is given and then, emphasis is placed on the distributed replicator dynamics. Using this control strategy a simple application example is introduced, and results of RTC using the MatSWMM run-off models (i.e., SWMM and the COM models) and its control module are presented.

\section{A. Population Dynamics}

This approach is presented by making an analogy with UDS or with a DWN. The UDS is mainly associated to the convergence topology shown in Figure 1a) as in [2], while the DWN is mainly associated to the divergence topology shown in Figure 1b) as in [11].

First of all, the analogy between elements in the population dynamics approach with elements in either USD or DWN is presented in Table I.

For instance, consider the system with the convergence topology shown in Figure 1a) with $m \in \mathbb{Z}_{+}$source reservoirs (strategy). The total flow through the system (population mass) 
TABLE I

EQUIVALENCE BETWEEN POPULATION DYNAMICS AND UDS/DWN

\begin{tabular}{lll}
\hline Population dynamics & UDS & DWN \\
\hline \hline Population & System & System \\
Strategy & Source reservoirs & Receptor reservoirs \\
Population mass & Total inflow to receptor reservoir & Total outflow source reservoir \\
Agent & Flow unit & Flow unit \\
Proportion of agents & Proportion of flow & Proportion of flow \\
Strategic distribution & Flow distribution in source reservoirs & Flow distribution in receptor reservoirs \\
Fitness of a strategy & Current volume & Available volume capacity \\
\hline
\end{tabular}

is denoted by $Q(t) \in \mathbb{R}_{+}$, which corresponds to the inflow of the receptor reservoir in the convergence topology. Hence, $Q(t)$ is composed of a large and finite amount of flow units (agents). Each flow-unit is assigned to an outflow of the source reservoirs, where the set of source reservoirs is denoted by $\mathcal{S}=\{1, \ldots, m\}$.

Regarding the divergence topology shown in Figure 1b), there are $m \in \mathbb{Z}_{+}$receptor reservoirs (strategy). Similarly, $Q(t) \in \mathbb{R}_{+}$corresponds to the outflow of the source reservoir in the divergence topology. Each flow-unit is assigned to an inflow of the receptor reservoirs, where the set of receptor reservoirs is denoted by $\mathcal{S}=\{1, \ldots, m\}$.

For both topologies, the scalar $p_{i}(t)$ is the proportion of flow units assigned to each outflow source reservoir $i \in \mathcal{S}$ as a percentage, i.e., the outflow for the $i^{\text {th }}$ reservoir is given by $p_{i}(t) Q(t)$. The vector $\mathbf{p}(t) \in \mathbb{R}_{+}^{m}$ is the flow proportion distribution involving the $m$ reservoirs. The set of the possible distributions of flow is given by a simplex $\Delta=\left\{\mathbf{p}(t) \in \mathbb{R}_{+}^{m}: \mathbf{p}(t)^{\top} \mathbb{1}_{m}=1\right\}$, and the interior of the set of the possible distributions of flow is given by the set $\tilde{\Delta}=\left\{\mathbf{p}(t) \in \mathbb{R}_{++}^{m}: \mathbf{p}(t)^{\top} \mathbb{1}_{m}=1\right\}$. Finally, the tangent space of the set of possible distributions of flow is defined as $\mathrm{T} \Delta=\left\{\mathbf{z}(t) \in \mathbb{R}^{m}: \mathbf{z}(t)^{\top} \mathbb{1}_{m}=0\right\}$.

For the convergence topology, each flow unit is assigned to each reservoir $i \in \mathcal{S}$ depending on the current volume, which is described by a function denoted by $F_{i}(\mathbf{p}(t))$. Then, more outflow is assigned to those reservoirs close to be filled up impeding overflows. In contrast, for the divergence topology, each flow unit is assigned to each reservoir $i \in \mathcal{S}$ depending on the current volume capacity, which is described by a function denoted by $F_{i}(\mathbf{p}(t))$. Therefore, less inflow is assigned to those reservoirs close to be filled up.

The design of the population-dynamics based controllers are given by the proper selection of the fitness functions that define the incentives for the proportion of agents. Furthermore, it is necessary that the fitness functions satisfy conditions to obtain a stable population game, i.e., the fitness functions should be decreasing with respect to the proportion of agents [12]. Notice that for the convergence topology (see Figure 2a)), the fitness functions can be selected increasing with respect to the current volumes [2]. When a proportion of agents is increased, then it is expected that the corresponding volume decreases, and consequently fitness function decreases with respect to the proportion of agents. In contrast, for the divergence topology (see Figure 1b)), the fitness functions can be selected decreasing with respect to the current value, e.g., the error with respect to the maximum capacity volume [11]. When a proportion of agents is increased, then it is expected that the corresponding volume increases, and consequently fitness function decreases with respect to the proportion of agents.

\section{B. Distributed replicator dynamics}

The traditional replicator dynamics equation is one of the six fundamental population dynamics [12], and it requires full information (i.e., all strategies associates to reservoirs need information about all other reservoir state in order to evolve). However, the distributed replicator dynamics are proposed in [10], and afterwards they are deduced in [1] from a local revision protocol that only needs partial information. Due to the fact that only local information is needed, then there is a undirected non-complete connected graph describing possible interaction denoted by $\mathcal{G}=(\mathcal{V}, \mathcal{E})$, where $\mathcal{V}$ is the set of nodes, which represents the reservoirs, and $\mathcal{E} \subset\{(i, j): i, j \in \mathcal{V}\}$ is the set of links representing the information sharing within the system. The distributed replicator dynamics are given by,

$\frac{d p_{i}(t)}{d t}=p_{i}(t)\left(F_{i}(\mathbf{p}(t)) \sum_{j \in \mathcal{N}_{i}} p_{j}(t)-\sum_{j \in \mathcal{N}_{i}} p_{j}(t) F_{j}(\mathbf{p}(t))\right)$,

for all $i \in \mathcal{S}$, where $\mathcal{N}_{i}=\{j:(i, j) \in \mathcal{E}\}$ is the set of neighbors of the node $i \in \mathcal{V}$. Notice that $i \notin \mathcal{N}_{i}$, and that $\mathcal{N}_{i} \neq \emptyset$, for all $i \in \mathcal{V}$ since $\mathcal{G}$ is connected.

\section{Application example}

The application example is related to the allocation of flows in a network where the convergence topology prevails. This structure is common in systems where the drainage flows lead to a single treatment plant (see Figure 2b). The system is composed by four subcatchments and a network of channels that converge to an outfall. The model can be divided in two main branches, which are determined by the nodes where several flows converge. In the first branch, there are two sections converging to a single node, while there are three in the second one. With that in mind, an equivalent model of the system can be obtained as done in [2], using partitions (see Figure 1b).

The disturbances of the system are related to direct runoff hydrographs in header partitions, i.e., channels that do not 
receive the flows from other receptor structures, such as $T_{1}$, $T_{2}, T_{3}$, and $T_{4}$ (see Figure $2 \mathrm{~b}$ ). The hydrographs are generated by SWMM with the Horton rainfall-runoff infiltration model [6] (see Figure 3).

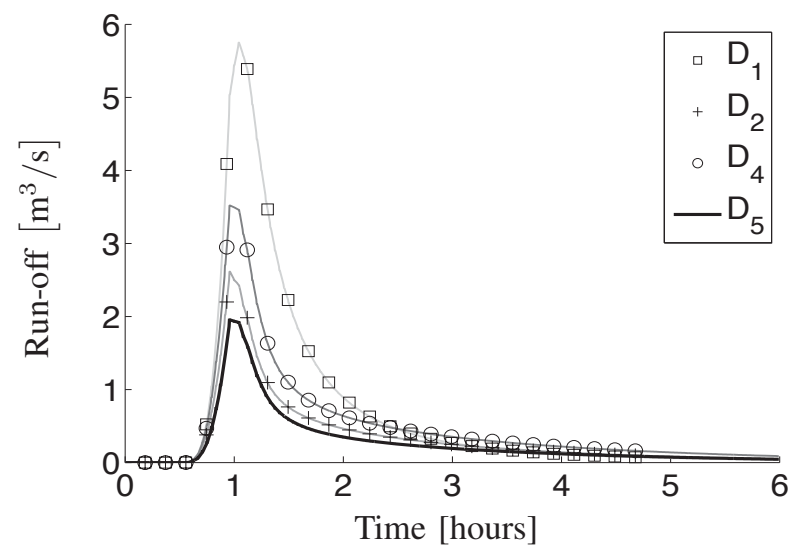

Fig. 3. Direct run-off hydrographs - disturbances $D_{i}$ for $i=\{1,2,4,5\}$.

The rain scenario produces flooding downstream, and some of the structures upstream are not properly filled, so the can be better managed, i.e., the capacity of the channels can be controlled to retain water upstream in order to prevent flooding downstream, using retention gates as actuators. With that in mind, the control objective is to maximize the filling rate of the channels, using efficiently the available space in order to prevent flooding downstream.

\section{RESUlts AND DISCUSSION}

The results are obtained with a rain scenario designed to illustrate the consequences of flooding downstream (see Figure 3 ). The performance of the system is tested on the VT-based model and the SOM incorporated in SWMM using the SVE. Table II shows the total amount of flooding for the case study. When no control is used, the total overflow downstream is $611.88 \mathrm{~m}^{3}$ and the occupancy of the reservoirs upstream is nearly $30 \%$ of their total capacity (see Figure $4 a$ ). The capacity $C_{i}(t)$ of the $\mathrm{i}$-th reservoir is defined as the normalized current volume at the structure, i.e., $C_{i}(t)=V_{i}(t) / V_{i}^{\max }$. This fact means that the reservoirs are not optimally used since they could retain some run-off upstream to minimize the overflow downstream.

Likewise, the results with the COM are shown in Figures 4c) and 4f)). When the control strategy is applied to the COM there is no flooding and the reservoirs are better managed, retaining run-off upstream, easing the burden downstream. Despite these results, the behavior of the system is quite different when the SOM is used (see Figures 4b) and 4d)). Although the total overflow is slightly reduced with respect to the uncontrolled case, the reservoirs are not well managed and the upstream reservoirs remain underused.

Even though there is a significant reduction on the total overflow of the system when the SVE are used for simulation - nearly half of the total amount of flooding - the performance is still overcome by the performance on the COM. It is important to notice that the difference between the results obtained with control for the SOM and without control are almost imperceptible, except for the reduction in excess of capacity at the reservoir $T_{6}$. This is a consequence of designing the controller under the assumption that actuating a retention gate can significantly affect the way water is stored throughout a partition of the system (i.e., a set of channels). In fact, the movement of the gate has quite small influence over the nearest channel for this case study. That is why a complete reduction of flooding volumes is not achieved when the SOM is used.

TABLE II

TOTAL OVERFLOW IN THE CASE STUDY

\begin{tabular}{lc}
\hline Control case & Overflows $\left[\mathbf{m}^{3}\right]$ \\
\hline \hline Without Control & 611.88 \\
Control with SWMM & 343.92 \\
Control with COM & 0 \\
\hline
\end{tabular}

Additionally, there is a difference between the time responses in the SOM and the ones in the COM. The response in the VT-based model is faster, which facilitates the task for the controller. As shown in Figures 4c) and 4f), the run-off is almost fully evacuated after six hours, while the same does not happen in the SOM (see Figures 4b) and 4c)), where some capacity is still occupied at each reservoir. This might suggest that modeling a control strategy with the COM allows to reach the control objective of evacuating water from the system as fast as possible, by means of the control actions. However, it cannot be stated with certainty if so, since the VT-based model does not considerate acceleration effects.

Concisely, COMs are simplified representations that capture some of the significant parts of the dynamics of the system. However, some details may be lost during the modeling process. For instance, the VT-based model only considers the mass balance principle, because of this, important phenomena like velocity variations or backwater are not properly modeled. Thus, no controller is going to have the same performance with a COM as with the SOM, such as the one incorporated in MatSWMM. Moreover, if the control design principles are based on the dynamics of a simplified model, it is possible that the controller would not satisfy its objectives and the system may experiment malfunction.

\section{CONCLUSIONS AND FURTHER WORK}

This paper has presented the MatSWMM tool for the study of RTC in UDS. It has been shown that the enhanced functionality that MatSWMM adds to SWMM allows to detect issues related to transient flow in open channels, leading to successful design and implementation of control strategies. The framework is tested on a convergence network showing that the overflows on the systems can be minimized using a default control strategy that comes with MatSWMM, which is a model-free decentralized control based on population dynamics. The framework also provides the possibility of developing a COM, based on the VT-based modeling approach, which can be used with model-dependent control strategies, 


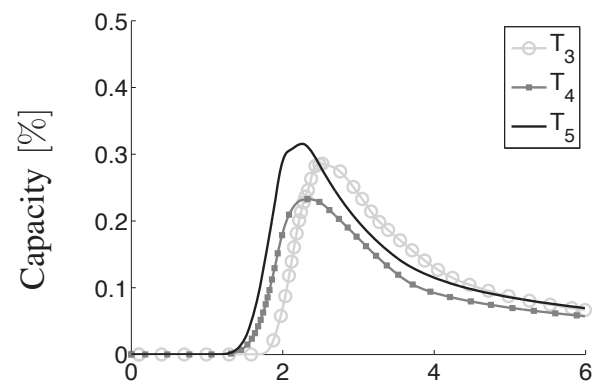

a)

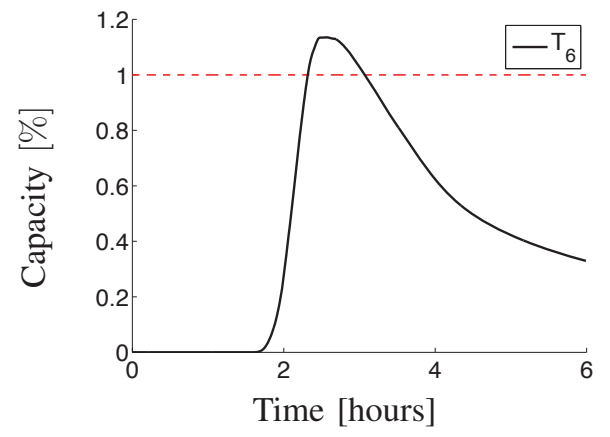

d)

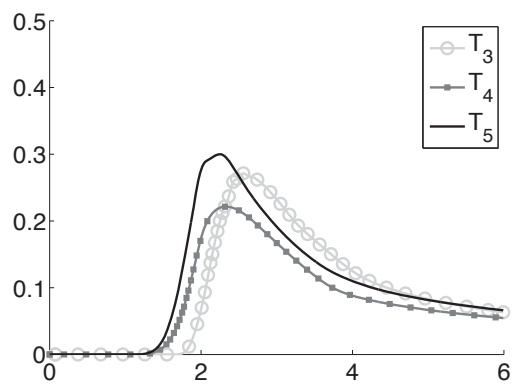

b)

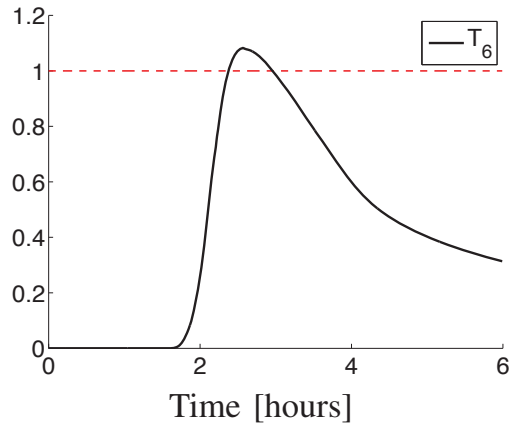

e)

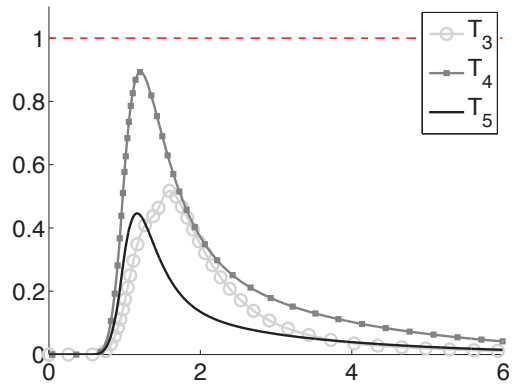

c)

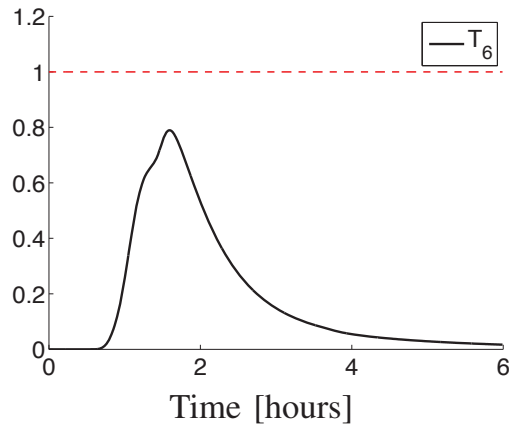

f)

Fig. 4. (a, d) capacity of reservoirs $T_{3}, T_{4}, T_{5}$ and $T_{6}$, when no control strategy is applied; (b, e) capacity of reservoirs $T_{3}, T_{4}, T_{5}$ and $T_{6}$, when the control strategy is applied to SWMM; (c, f) capacity of reservoirs $T_{3}, T_{4}, T_{5}$ and $T_{6}$, when the control strategy is applied to the COM.

such as model predictive control. Finally, it is shown that the use of simplified models, that are only based on the mass balance principle like the VT-based model, as a reference for the design of controllers in UDS, can lead to malfunction of the system.

As further work, it is possible to implement libraries associated to optimal positioning of actuators (e.g., retention and redirection gates). It is also suggested to work on the optimal sizing and positioning of storage elements into the network to better manage the run-off throughout the system and thus allowing smaller overflows. Finally, as MatSWMM provides a COM of the system, one could work on developing default control strategies for the tool that are model-dependant.

\section{ACKNOWLEDGMENT}

Authors would like to thank Mexichem (Colombia) for supporting this research through the project Drenaje Urbano y Cambio Climtico: hacia los sistemas de alcantarillado del futuro. Fase II. COLCIENCIAS 633/2013. Also, this work has been partially supported by the project ECOCIS (Ref. DPI2013-48243-C2-1-R). J. Barreiro-Gomez is partially supported by COLCIENCIAS-COLFUTURO and Agencia de Gestio d'Ajust Universitaris i de Recerca AGAUR.

\section{REFERENCES}

[1] J. Barreiro-Gomez, G. Obando, and N. Quijano. Distributed population dynamics: Optimization and control applications. IEEE Transactions on Systems, Man, and Cybernetics (Submitted), 2015.

[2] J. Barreiro-Gomez, G. Obando, G. Riaño Briceño, N. Quijano, and C. Ocampo Martinez. Decentralized control for urban drainage systems via population dynamics: Bogotá case study. In Proceedings of the 14th European Control Conference, Linz, Austria, 2015.
[3] D. Butler and M. Schütze. Integrating simulation models with a view to optimal control of urban wastewater systems. Environmental modelling \& software, 20(4):415-426, 2005.

[4] G. Cembrano, J. Quevedo, M. Salamero, V. Puig, J. Figueras, and J. Mart1. Optimal control of urban drainage systems. a case study. Control Engineering Practice, 12(1):1-9, 2004.

[5] L. A. García, E. Escobar, J. Barreiro-Gómez, N. Quijano, C. OcampoMartínez, and D. Téllez. On the modeling and real-time control of urban drainage systems: A survey. In Proceedings of the 11th International Conference on Hydroinformatics, 2014.

[6] W. C. Huber, L. A. Rossman, and R. Dickinson. EPA storm water management model SWMM 5.0. National Risk Management Research Laboratory, Office of Research and Development, US Environmental Protection Agency, 2010.

[7] J. Lin, S. Sedigh, and A. Miller. Towards integrated simulation of cyber-physical systems: a case study on intelligent water distribution. In Proceedings of the 8th IEEE International Conference on Dependable, Autonomic and Secure Computing, pages 690-695, Chengdu, China, 2009.

[8] A Messmer, M Schütze, and M Ogurek. A demonstration software tool for real time control of urban drainage systems. In Proceedings of the 11th international Conference on Urban Drainage (ICUD), Edinburgh, Scotland, UK, 2008.

[9] C. Ocampo-Martinez. Model Predictive Control of Wastewater Systems. Springer Verlag, 2010.

[10] A. Pantoja and N. Quijano. Distributed optimization using population dynamics with a local replicator equation. In Proceedings of the 51st IEEE Conference on Decision and Control (CDC), pages 3790-3795, Maui, Hawaii, USA, 2012.

[11] E. Ramirez-Llanos and N. Quijano. A population dynamics approach for the water distribution problem. International Journal of Control, 83(9):1947-1964, 2010.

[12] W. H. Sandholm. Population games and evolutionary dynamics. Economic learning and social evolution. Cambridge, Mass. MIT Press, 2010.

[13] V. Te Chow. Open channel hydraulics. McGraw-Hill Book Company, Inc; New York, 1959. 